\title{
Contemporary Indications in Breast Reconstruction
}

Editors

JIAN FARHADI

STEFAN O.P. HOFER

JAUME MASIA

\section{CLINICS IN \\ PLASTIC SURGERY}

www.plasticsurgery.theclinics.com

January 2018 • Volume 45 - Number 1 